\title{
Low-energy excitation spectrum of one-dimensional dipolar quantum gases
}

\author{
S. De Palo, ${ }^{1}$ E. Orignac, ${ }^{2,3}$ R. Citro, ${ }^{4,5}$ and M. L. Chiofalo ${ }^{6,7}$ \\ ${ }^{1}$ DEMOCRITOS INFM-CNR and Dipartimento di Fisica Teorica, Università di Trieste, I-34100 Trieste, Italy \\ ${ }^{2}$ Université de Lyon, Lyon, France \\ ${ }^{3}$ Laboratoire de Physique, École Normale Supérieure de Lyon, CNRS-UMR5672, Lyon, France \\ ${ }^{4}$ Dipartimento di Fisica "E. R. Caianiello" and CNISM, Università degli Studi di Salerno, I-84100 Salerno, Italy \\ ${ }^{5}$ Université Grenoble-I and CNRS, UMR5493, Grenoble, France \\ ${ }^{6}$ INFN, Dpt. of Mathematics and Faculty of Pharmacy, University of Pisa, I-56100 Pisa, Italy \\ ${ }^{7}$ Classe di Scienze, Scuola Normale Superiore, I-56100 Pisa, Italy
}

(Received 7 November 2007; revised manuscript received 21 May 2008; published 23 June 2008)

\begin{abstract}
We determine the excitation spectrum of a bosonic dipolar quantum gas in a one-dimensional geometry from the dynamical density-density correlation functions simulated by means of reptation quantum Monte Carlo techniques. The excitation energy is always vanishing at the first vector of the reciprocal lattice in the whole crossover from the liquidlike at low density to the quasiordered state at high density, demonstrating the absence of a roton minimum and thus the absence of superfluidity in the Landau sense. Gaps at higher reciprocal-lattice vectors are seen to progressively close with increasing density, while the quantum state evolves into a quasiperiodic structure. The simulational data together with the uncertainty-principle inequality also provide a rigorous proof of the absence of long-range order in such a superstrongly correlated system. Our conclusions confirm that the dipolar gas is in a Luttinger-liquid state and that the Feynman spectrum inferred from the static structure factor yields in most cases an inaccurate description. The connection with ongoing experiments is also discussed.
\end{abstract}

DOI: 10.1103/PhysRevB.77.212101

PACS number(s): 03.75.Kk, 71.10.Pm, 02.70.Ss, 03.75.Hh

Ultracold quantum gases with dipolar interactions are currently being produced in laboratory, where atomic ${ }^{52} \mathrm{Cr}$ atoms have been Bose condensed, ${ }^{1}$ following earlier theoretical predictions. ${ }^{2}$ Experiments have been suggested, ${ }^{3}$ aimed to produce molecular gases with large dipolar strengths, and a few laboratories worldwide are working along these lines. In fact, dipolar quantum gases are emerging as competitive realizations of quantum devices ${ }^{4}$ and as a laboratory for investigating strongly correlated regimes ${ }^{5,6}$ and novel quantum phases, ${ }^{7}$ in which quantum fluctuations are enhanced by exploiting techniques acquired for an accurate manipulation of atomic gases. These include the possibility of lowering the temperature, of tuning the interactions in both their longrange tail $^{8}$ and in the strength of their short-range $\operatorname{part}^{9}$ by means of the Fano-Feshbach mechanism ${ }^{10}$ to let the dipolar character ${ }^{11}$ emerge, and of reducing the dimensionality down to one dimension, as already performed in other systems. ${ }^{12}$

One-dimensional (1D) quantum gases are naturally inclined to be strongly correlated. ${ }^{13,14}$ We have more recently predicted that dipolar bosonic quantum gases confined in quasi-1D geometries can reach correlation regimes well beyond those of the (already strongly correlated) TonksGirardeau (TG) gas, ${ }^{15}$ crossing over to a dipolar-densitywave (DDW) (Ref. 16) state at very large densities $n$ on the scale of the potential range, where the atoms arrange into an ordered state regularly spaced by $1 / n$. By a back-to-back comparison with reptation quantum Monte Carlo (RQMC) (Ref. 17) simulational data, we have shown that at the level of the static structure factor, the crossover can be described by a Luttinger-liquid (LL) theory with exponent $K<1$ continuously decreasing from $K=1 \quad n r_{0} \rightarrow 0$ to $K \rightarrow 0$ as $n r_{0} \rightarrow \infty$. Finally, we have predicted the corresponding signatures in the collective excitations of the trapped gas. ${ }^{18}$

Beyond the evidence emerging from the static structure of the fluid, a clear-cut demonstration of Luttinger behavior re- quires further understanding of the excitations in the homogeneous dipolar gas. In particular, answers to two relevant questions are not obvious from the beginning. First, whether rotonlike excitations may show up in the dipolar gas at finite wave vectors. Second, whether the quantum fluctuations of the phonon field prevent the existence of long-range order at large densities, namely, whether the crystal order parameter vanishes in the thermodynamic limit, as first discussed by Bogoliubov. ${ }^{19}$ In fact, exploiting the uncertainty principle instead of the Bogoliubov inequality, Pitaevskii and Stringari ${ }^{20}$ worked out an extension of the Hohenberg-Mermin-Wagner theorem, ${ }^{21}$ which yields more accurate upper bounds to the size of the order parameter at zero temperature, where the quantum fluctations dominate. When applied to specific systems, the inequality may allow us to rule out the existence of long-range order, as in the case of, e.g., 1D antiferromagnets and crystals. ${ }^{20}$ Both questions above would have a definite answer if the system were in a LL state, for which there is no long-range order nor roton minimum.

We find that this is the case by means of a finite-size analysis of the low-energy excitation spectrum, as extracted from RQMC density-density correlation function in imaginary time at the reciprocal-lattice vector $G_{m} / n=2 \pi m, m$ $=1, \ldots 4$. The evolution of quasi-long-range order from the TG to the DDW state emerges as a progressive closing of the gaps in the excitation spectrum with increasing the order $m$. By the same token, we show the absence of a roton minimum at $2 \pi$ in the whole crossover and that dynamical effects play a significant role in building the Luttinger state. Our results, analyzed by means of the uncertainty-principle inequality ${ }^{20}$ also rule out the existence of long-range order in this superstrongly correlated quantum gas and confirm that the 1D dipolar gas is in a LL state.

We model the 1D dipolar Bose gas by considering $N$ atoms with mass $M$ and permanent dipoles moments arranged 
along and orthogonal to a line, yielding purely repulsive interactions. The Hamiltonian is

$$
H=-\frac{1}{r_{s}^{2}} \sum_{i} \frac{\partial^{2}}{\partial x_{i}^{2}}+\frac{1}{r_{s}^{3}} \sum_{i<j} \frac{1}{\left|x_{i}-x_{j}\right|^{3}}
$$

in effective Rydberg units $R y^{*}=\hbar^{2} /\left(2 M r_{0}^{2}\right)$. The effective Bohr radius $r_{0} \equiv M C_{d d} /\left(2 \pi \hbar^{2}\right)$ is expressed in terms of the interaction strength $C_{d d}=\mu_{0} \mu_{d}^{2}$ for magnetic and $C_{d d}=d^{2} / \epsilon_{0}$ for electric dipoles. ${ }^{22}$ The dimensionless parameter $r_{s}$ $=1 /\left(n r_{0}\right)$ determines the interacting regime in terms of $r_{0}$ and of the linear density $n$. Since the potential-to-kineticenergy ratio scales as $1 / r_{s}=n r_{0}$, large densities yield strong correlations.

We determine the excitation spectrum while the parameter $n r_{0}$ spans the whole crossover from the TG to the DDW state from the analysis of the imaginary-time density-density correlation function: $F(q, \tau)=\left\langle\rho_{q}(\tau) \rho_{q}^{\dagger}(0)\right\rangle / N$, where $\rho_{q}$ $=\sum_{i}^{N} \exp \left[-i q \cdot x_{i}(\tau)\right]$ is the density-fluctuation operator at wave vector $q$ and imaginary time $\tau$, the sum spanning over the $N$ particles located at position $x_{i}$. To compute this quantity we resort to the RQMC technique, ${ }^{17,23}$ which is in essence a path-integral method at zero temperature, where the ground-state distribution is directly sampled in the internal part of the path. Thus, the computation of the imaginarytime-correlation functions is conceptually straightforward and practically easy.

We use a trial wave function that is a product of two-body Jastrow factors $\psi_{\text {trial }}=\prod_{i<j} e^{u\left(\left|x_{i}-x_{j}\right|\right)}$. As we are interested in long-range behavior, we actually use the LL expression,

$$
\psi_{\text {trial }}(R) \propto \prod_{i<j}\left|\sin \frac{\pi}{L}\left(x_{i}-x_{j}\right)\right|^{1 / K},
$$

which in the low-density limit, where $K=1,5$ recovers the wave-function of spinless noninteracting fermions. Different choices of the wave functions, such as the product of Gaussians centered on the lattice sites $R_{m}=m n^{-1}$, result in different time-step extrapolations for the energy, but eventually lead to negligible differences in the computation of the imaginary-time-correlation functions.

We perform simulations for different values of the number $N$ of bosons in a square box with periodic boundary conditions, namely, $N=40,40,60,80$, and 100 , reaching in selected cases $N=200$. We reduce finite-size effects by summing the interactions over ten simulation boxes. Groundstate quantities of interest are computed at long enough values of the projection time for which the energies saturate and with a small enough time step to make the results independent of the time step itself. The energy per particle as a function of $n r_{0}$ has already been provided in (Ref. 18) together with an accurate analytical form for it. This form recovers the known TG and DDW limiting behaviors and can be used for further applications.

The energy of the low-lying excitations are extracted after exploiting the Laplace transformation connecting $F(q, \tau)$ to the dynamical structure factor, that is, $F(q, \tau)$ $=\int_{0}^{\infty} d \omega \exp (-\omega \tau) S(q, \omega)$. In practice, specific choices of $S(q, \omega)$ are used, which introduce a controllable level of approximation in the method. We assume as suitable form for the dynamical structure factor at zero temperature,

$$
S(q, \omega)=\sum_{i}^{\alpha} A_{i}(q) \delta\left(\omega-\omega_{i}\right),
$$

and within this choice $F(q, \tau)=\sum_{i=1, \alpha} A_{i}(q) e^{\omega_{i}(q) \tau}$, where $\alpha$ is the number of excitation modes needed to yield the best $\chi^{2}$ (chi-square) in the fitting procedure and depends on the values of $q$ and $n r_{0}$.

Before proceeding further, we notice that the specific choice (3) implies an approximation. In order to control the robustness of such a choice, we have considered different forms for $S(q, \omega)$. In particular, we have (i) replaced the delta functions in Eq. (3) with sharp peaked Gaussians and (ii) assumed the form $S(q, \omega)=A\left[\omega-\omega_{\min }(q)\right]^{a}$ for the lowest mode $\omega_{i}(q)$ for $\omega>\omega_{\min }$ and 0 elsewhere, leading to $F(q, \tau)=A \Gamma(a+1) \exp \left[-\tau \omega_{\min }(q)\right] \tau^{(-a-1)}$ valid in the long $\tau$ limit. In case (i), we have found a worsened quality of the fits which has prevented a systematic analysis, while in case (ii) we have found insignificant changes in the qualitative and quantitative picture as obtained from Eq. (3).

We now turn back to analyze the dependence of the number of modes $\alpha$ on $q$ and $n r_{0}$. For low enough wave vectors $q$ indeed, the Feynman approximation corresponding to $\alpha$ $=1$ is the best choice at all densities. While at $q=2 \pi$, a multimode description with $\alpha \geq 2$ is often needed as the density is lowered, and in the extreme low-density limit-eventually mapping into the noninteracting Fermi gas-two modes are enough to fit $F(q, \tau)$. Best $\chi^{2}$ values also correspond to best degrees to which the $f$-sum rule $\int d \omega \omega S(q, \omega)=2 \pi n q^{2}$ is satisfied. In the very high-density limit a single-mode fit of $F(q=2 \pi, \tau)$ has $\chi^{2} \simeq 0.003$, while the $f$-sum rule is satisfied within $2 \%$. More modes are progressively needed for higher momenta, such as $q=4 \pi$ and $q=6 \pi$, at all densities. In the intermediate and low-density regime, such as $n r_{0}=1$ and 0.1 , the Feynman approximation yields a $\chi^{2}$ of the order of 50 in the most favorable cases, while addition of a second and third mode allows us to improve the quality of the fit up to $\chi^{2} \approx 1$ and $\chi^{2} \approx 0.1$. On increasing the number of modes, the $f$-sum rule is fulfilled to a progressively better accuracy, passing from a $70 \%$ level with one mode to $30 \%$ with two and $10 \%$ with three modes. In the extreme low-density limit, $\chi^{2}$ values $\chi^{2} \approx 0.02$ and $f$-sum rule accuracies $5 \%$ are obtained with two modes, the quality of the fit not improving after adding more modes. In Fig. 1 the typical behavior of the $F(q, \tau)$ is displayed, showing how the quality of the fit improves while adding modes.

The emerging structure of the excitations is consistent with the weights expected for the so-called type-I and type-II modes of the Lieb-Liniger gas for 1D Bose systems. ${ }^{24}$ In the long $\tau$ limit of our RQMC simulations however, the behavior of $F(q, \tau)$ will be dominated by the lower branch, i.e., the type II excitations, as the upper branch has a much faster exponential decay.

Figure 2 displays the resulting lowest excitation energies $\omega(q)$, at $N=40$, for $n r_{0}=1,10$, and 1000. Despite finite-size effects, the overall qualitative behavior is already clear. At low $q$ values, where the Feynman approximation is sufficient, the phonon softens while the density decreases and at $2 \pi$, where the number of modes needed to fit $F(q, \tau)$ are 


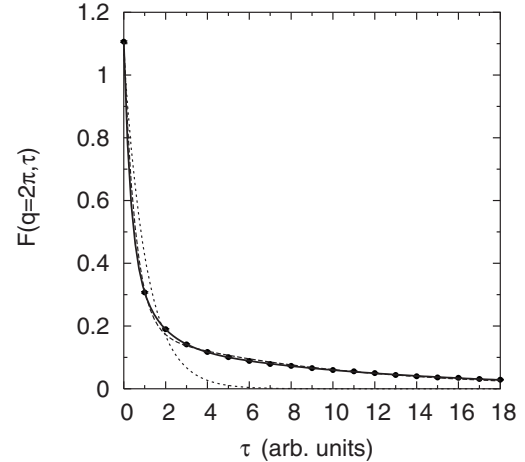

FIG. 1. $F(q, \tau)$ for $n r_{0}=0.1$ and $N=100$. Symbols: RQMC data (the error bars are not visible on this scale). Curves: fits to the RQMC data after including additional modes, which is $\alpha=1$ (dotted line), 2 (dashed), and 3 (solid line) modes. Adding a fourth mode did not improve the quality of the fit. The reduced $\chi^{2}$ changed from 1000 for $\alpha=1$ to 0.2 for $\alpha=3$.

$\alpha=1$ at $n r_{0}=1000$ and $\alpha=3$ for $n r_{0}=1,10$, the gap seems to be always closed at all densities.

As shown in the inset by the dotted curve, even at $n r_{0}$ $=1000$ the excitation spectrum (solid line) is very different from what could be obtained by replicating the portion from $q=0$ to $q=2 \pi$ (dotted line), indicating a nonperiodic structure. The dashed line represents $\omega(q)$ as obtained in the Feynman approximation $\omega(q)=\hbar^{2} q^{2} /[2 m S(q)]$, which provides only an upper bound. As expected, the range of validity of this approximation, that is, $q / n \rightarrow 0$, shrinks while lowering the density or increasing $q$.

A quantitatively reliable measure of the gap sizes requires an accurate size effect analysis. Figure 3 displays the $1 / N$ scaling of $\omega(q / n=2 \pi)$ for $n r_{0}=0.01,0.1,1,10$, and 1000. In all cases the finite-size lowest excitations energies yield a linear scaling $\omega_{N}(q=2 \pi)=c\left(n r_{0}\right) / N$ with the slope $c\left(n r_{0}\right)$ being an increasing function of $n r_{0}$. We see that the gap is closed at all densities, demonstrating the absence of a roton minimum. The Feynman approximation is able to reproduce the gap closure only in the high-density limit (see the inset of Fig. 3), while at the intermediate-to-low densities at least $\alpha$ $=3$ modes are needed to get a vanishing gap. ${ }^{25}$ Breakdown of the Feynman approximation for low and medium density is caused by the absence of coherent density excitations at $q$ $=2 \pi$. Indeed, the Feynman approximation assumes from the start that a single coherent mode accounts for most of the spectral weight. As the spectrum of density excitations in a LL at $q=2 \pi$ is incoherent, ${ }^{24,26}$ the averaging of the full spectral weight of the LL over a single mode overestimates the mode energy and leads to qualitatively different conclusions. A similar multimode analysis performed at $q=2 \pi m, m>2$ shows the existence of open gaps, which progressively close while the quasi-ordered state is approached.

Using these results, we can derive a strict upper bound for the order parameter of the solid,

$$
\rho_{q}=N^{-1}\left\langle\sum_{m} \exp \left(i G \cdot x_{m}\right)\right\rangle
$$

with $G$ as a vector of the reciprocal lattice and rigorously test the qualitative conclusions from the inset of Fig. 2, namely, that no long-range order may exist in our $1 \mathrm{D}$ dipolar quan- tum gas. We closely follow the derivation of Pitaevskii and Stringari. ${ }^{20}$ By applying the uncertainty-principle inequality

$$
\left\langle A^{\dagger}, A\right\rangle\left\langle B^{\dagger}, B\right\rangle \geq\left|\left\langle\left[A^{\dagger}, B\right]\right\rangle\right|^{2}
$$

to the operators $A=\hat{\rho}_{q+G}$ and $B=\partial \hat{\rho}_{q} / \partial t$, one has

$$
\left.S(q+G) \int d \omega \omega^{2} S(q, \omega) \geq \frac{1}{4 m^{2}} \rho_{G}^{2}\left(q \cdot(q+G)^{2}\right)\right) .
$$

From the RQMC data we know that as $q \rightarrow 0$, $S(q+G) \rightarrow|q|^{2 K-1}$, while the second moment of $S(q, \omega)$ vanishes as $|q|^{3}$. Thus, the order parameter in the longwavelength limit vanishes as $\rho_{G}^{2} \leq q^{\min (2 K, 1)}$ with $K \geq 0$. Thus no long-ranger order may exist unless $K=0$, which is however the limit of infinite density.

These results can be analyzed within the LL theory. We want to calculate the imaginary-time $\tau$ correlation function $\tilde{F}(x, \tau)=\left\langle T_{\tau} e^{i 2 \phi(x, \tau)} e^{-2 i \phi(0,0)}\right\rangle$ on a finite-size system of length $L$. It is known ${ }^{26}$ from bosonization that

$$
\tilde{F}(x, \tau)=\frac{\left(\frac{\pi \alpha}{L}\right)^{2 K}}{\left[\sinh ^{2}\left(\frac{\pi u \tau}{L}\right)+\sin ^{2}\left(\frac{\pi x}{L}\right)\right]^{K}} .
$$

This expression is valid in the long-time (low-energy) limit $u \tau \gg \alpha$, where $\alpha$ is now a short-distance cutoff of the order of $n^{-1}, u$ is the velocity of the excitations, and $K$ the Luttinger exponent. After Fourier transforming $\widetilde{F}(x, \tau)$ in $q$ space with $q=2 \pi j / L$, we get

$$
\begin{aligned}
F(q, \tau)= & \left(\frac{\pi \alpha}{L}\right)^{2 K} L 2^{2 K+1-j} \frac{e^{-[(2 \pi u \tau) / L](K+j)}}{\left(1+e^{-(2 \pi u \tau) / L}\right)^{K+j}}[\Gamma(j+K)] \\
& \times[\Gamma(K) \Gamma(j+1)]^{-1}{ }_{2} F_{1}\left(K+j, K ; j+1 ; e^{-(2 \pi u \tau) / L}\right),
\end{aligned}
$$

where ${ }_{2} F_{1}$ and $\Gamma$ are the hypergeometric and Euler functions.

Using an ansatz $e^{-\tau \omega_{1}(q)}$ to fit the long-time behavior $F(q, \tau \rightarrow \infty) \sim e^{-[(2 \pi u \tau) / L](K+j)}$, we get

$$
\omega(q)=\frac{2 \pi u K}{L}+u|q|,
$$

where we have used the even parity of the response function. Thus, there should be no roton gap at $q=2 \pi$ in the infinite size limit. For finite size, an apparent roton gap (vanishing as $1 / L)$ can be seen. This gap can be traced to the zero mode contribution to the correlation functions. All the fits to the RQMC data presented in Figs. 2 and 3 reproduce remarkably well this $1 / L$ scaling $^{27}$ and are consistent with our previous findings on the density dependence of Luttinger- $K$ exponent $K(n){ }^{5}$

In conclusion, the analysis of the RQMC simulational data neatly leads to two main conclusions, namely, that there are no roton excitations appearing at the first star of the reciprocal lattice and that no long-range order may exist in the whole crossover from the TG gas at low density to the quasiordered DDW state at high densities. The absence of a roton gap implies the absence of superfluidity in this system, according to the Landau criterion. The RQMC data analysis is in remarkable agreement with what expected for a superstrongly correlated LL state. The realization of 1D dipolar 


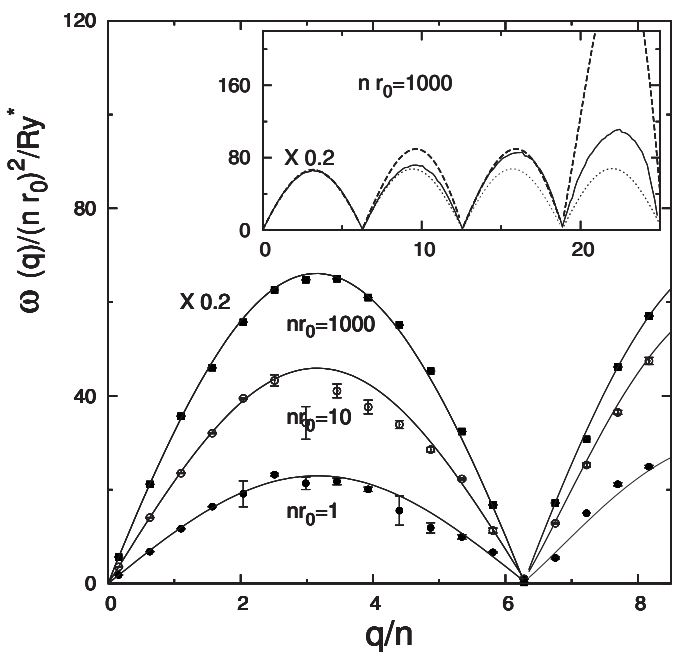

FIG. 2. Lowest excitation energies $\omega(q)$ in $R y^{*}$ units and scaled by $\left(n r_{0}\right)^{2}$ for a dipolar gas with $N=40$ and different values of $n r_{0}$ $=1,10$, and 1000 as in the legend. The symbols with error bars are energies extracted using Eq. (3); the solid line is a guide to the eye. The curve at $n r_{0}=1000$ is depressed by a factor of 5 for graphical reasons. Inset: zoom on the $\omega(q)$ at $n r_{0}=1000$ up to $q / n=8 \pi$ for different $F(q, \tau)$ models: multimode model (3) (solid) and Feynman (dashed) approximation. Dotted line: periodic replica of the first bump.

quantum (molecular) gases in the TG to the DDW regime is within reach of current experimental efforts ${ }^{5,28}$ and thus our predictions on the excitation spectrum, in particular, on the absence of the roton minimum and of superfluidity, can be tested in future experiments by means of, e.g., Bragg spectroscopy techniques. ${ }^{29}$

${ }^{1}$ J. Stuhler et al., Phys. Rev. Lett. 95, 150406 (2005); A. Griesmaier et al., ibid. 94, 160401 (2005).

${ }^{2}$ L. Santos et al., Phys. Rev. Lett. 85, 1791 (2000); K. Góral et al., Phys. Rev. A 61, 051601(R) (2000).

${ }^{3}$ H. P. Büchler et al., Phys. Rev. Lett. 98, 060404 (2007).

${ }^{4}$ P. Rabl and P. Zoller, Phys. Rev. A 76, 042308 (2007).

${ }^{5}$ R. Citro et al., Phys. Rev. A 75, 051602(R) (2007).

${ }^{6}$ A. S. Arkhipov et al., JETP Lett. 82, 39 (2005); G. E. Astrakharchik and Y. E. Lozovik, Phys. Rev. A 77, 013404 (2008).

${ }^{7}$ K. Góral et al., Phys. Rev. Lett. 88, 170406 (2002); H. Pu et al., ibid. 87, 140405 (2001).

${ }^{8}$ S. Giovanazzi et al., Phys. Rev. Lett. 89, 130401 (2002).

${ }^{9}$ T. Lahaye, T. Koch, B. Fröhlich, M. Fattori, J. Metz, A. Griesmaier, S. Giovanazzi, and T. Pfau, Nature (London) 448, 672 (2007).

${ }^{10}$ H. Feshbach, Ann. Phys. 5, 357 (1958); U. Fano, Phys. Rev. 124, 1866 (1961).

${ }^{11}$ S. Ronen et al., Phys. Rev. A 74, 033611 (2006).

${ }^{12}$ B. Paredes et al., Nature (London) 429, 277 (2004); T. Kinoshita et al., Science 305, 5687 (2004).

${ }^{13}$ T. Giamarchi, Quantum Physics in One Dimension (Oxford University Press, Oxford, 2004).

${ }^{14}$ Thus, 1D dipolar quantum gases are amenable to a variety of interesting effects, as e.g., spin-charge separation. See, e.g., A. Kleine et al., Phys. Rev. A 77, 013607 (2008).

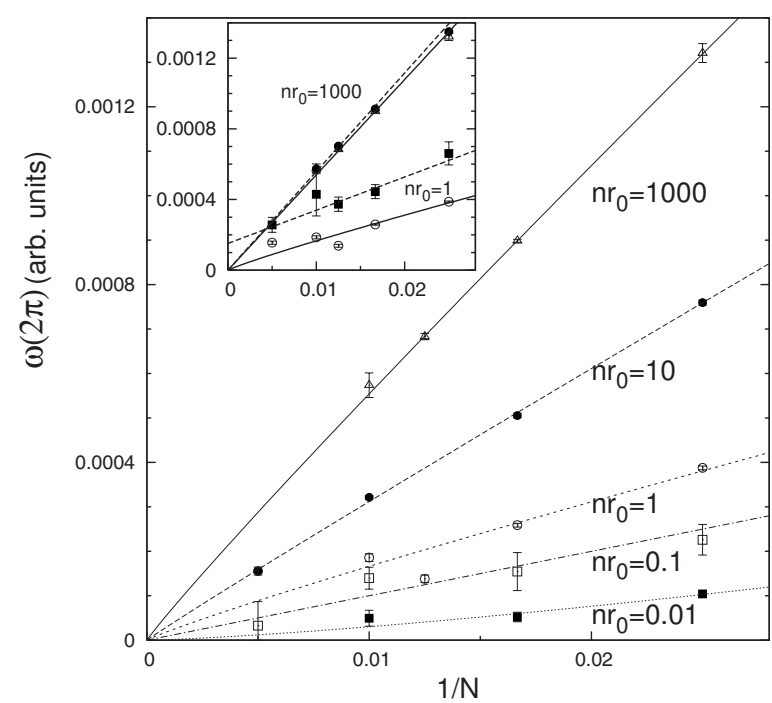

FIG. 3. $1 / N$ scaling of $\omega(q=2 \pi)$ in arbitrary units at $n r_{0}=0.01$, $0.1,1,10$, and 1000 as in the legend. Symbols with error bars: RQMC data. Solid lines: fit to the data. Inset: zoom of the RQMC data and related fits for the cases with $n r_{0}=1$ and 1000. Filled symbols and dashed lines: Feynman relation (single-mode approximation). Open symbols and solid lines: multimode analysis yielding best $\chi^{2}$ with $\alpha=1$ for the highest density case $\alpha=3$ or 4 for the other cases.

We are especially grateful to the Scuola Normale Superiore for providing ideal conditions for the realization of large parts of this work and to G. La Rocca for interesting discussions and support. We also thank S. Stringari and L. Pitaevskii for pointing out the use of the uncertaintyprinciple inequality.
${ }^{15}$ M. Girardeau, J. Math. Phys. 1, 516 (1960).

${ }^{16}$ By dipolar-density wave, we mean a quasiordered state very much analogous to a charge-density wave.

${ }^{17}$ S. Baroni and S. Moroni, Phys. Rev. Lett. 82, 4745 (1999).

${ }^{18}$ P. Pedri et al., Phys. Rev. A 77, 015601 (2008).

${ }^{19}$ N. N. Bogoliubov, Quasi-Expectation Values in Problems of Statistical Mechanics (Gordon and Breach, New York, 1961).

${ }^{20}$ L. Pitaevskii and S. Stringari, J. Low Temp. Phys. 85, 377 (1991).

${ }^{21}$ N. D. Mermin and H. Wagner, Phys. Rev. Lett. 17, 1133 (1966); P. C. Hohenberg, Phys. Rev. 158, 383 (1967).

${ }^{22} \mu_{d}$ and $d$ are the magnetic and electric-dipole moments and $\mu_{0}$ and $\varepsilon_{0}$ are the vacuum permittivities.

${ }^{23}$ S. De Palo et al., Phys. Rev. B 69, 035109 (2004).

${ }^{24}$ E. H. Lieb, Phys. Rev. 130, 1616 (1963); G. E. Astrakharchik and L. P. Pitaevskii, Phys. Rev. A 70, 013608 (2004); J. S. Caux and P. Calabrese, ibid. 74, 031605(R) (2006); M. Khodas et al., Phys. Rev. Lett. 99, 110405 (2007).

${ }^{25}$ Adding more modes can improve the quality of the fit yet leading to the same qualitative results.

${ }^{26}$ T. Giamarchi, Quantum Physics in One Dimension (Oxford University Press, Oxford, 2004).

${ }^{27}$ At fixed density, $1 / N$ and $1 / L$ scaling are equivalent.

${ }^{28}$ R. Citro, S. D. Palo, P. Pedri, E. Orignac, and M. L. Chiofalo, New J. Phys. 10, 045011 (2008).

${ }^{29}$ D. M. Stamper-Kurn et al., Phys. Rev. Lett. 83, 2876 (1999). 